\title{
Physikalische Therapie: Neuroplastizität
}

\author{
Jean-Michel Jeannin
}

Basel, Schweiz

\section{Einleitung}

Lange Zeit galt das Dogma, dass sich Nervenzellen beim Erwachsenen nicht mehr teilen und die Gehirnstrukturen sich nicht mehr entwickeln können. Mittlerweile ist dieses Dogma widerlegt [1]. So wurde im Zusammenhang mit der Erforschung von Demenzkrankheiten untersucht, ob Interventionen im Sinn von Training und Anwendungen von Behandlungen der physikalischen Therapie das Fortschreiten einer Demenzerkrankung verzögern können. Ausgehend von einem Zerfall von Strukturen des Gehirns als Ursache für Demenzerkrankungen müsste eine wirksame Behandlung ihrerseits einen Einfluss auf diesen Verfall haben. Der vorangehende Beitrag befasste sich mit den molekularen Mechanismen, die für die zentralnervösen Effekte einer physikalischen Therapie verantwortlich sind [2]. Der vorliegende Beitrag thematisiert nun die anatomischen Aspekte, wobei auch Ergebnisse und Aspekte der Entwicklungsbiologie und -psychologie berücksichtigt werden (Abb. 1-3).

Abb. 1. Soziale Interaktionen sind wichtig für die Gesundheit (Gibraltar, 2017).

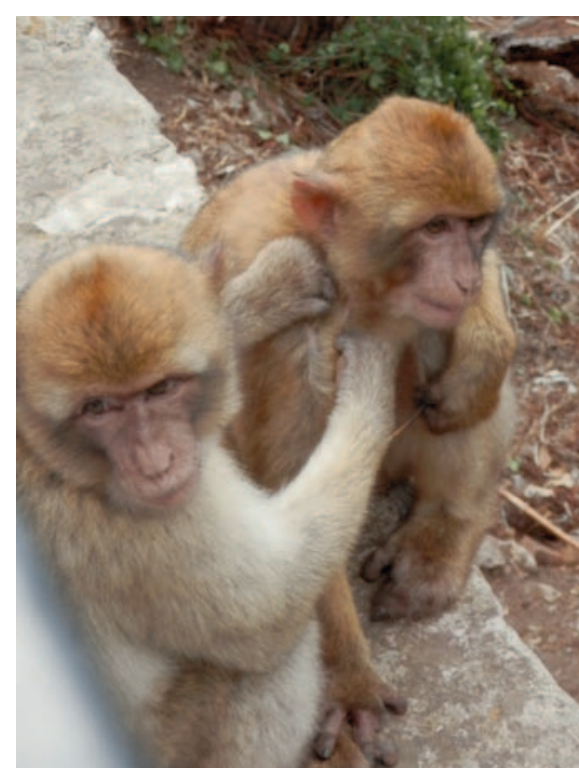

(c) 2017 S. Karger GmbH, Freiburg

Accessible online at:

www.karger.com/szg

\section{Neuroplastizität}

Der Begriff «Neuroplastizität» bezeichnet die Fähigkeit des Gehirns, seine Strukturen und Funktionen im Verlauf des Lebens eines Individuums den Anforderungen anzupassen. Der Begriff ist wissenschaftsgeschichtlich relativ alt. Die Erkenntnis, dass auch das Gehirn von Erwachsenen plastisch ist, ist jedoch sehr jung [3]. Die Erforschung der Neuroplastizität wurde von der in der Einleitung erwähnten Erforschung der Demenz stark befördert. Für die Diskussion des Themas ist es zweckmässig, die Neuroplastizität in der Entwicklung des Individuums von der des Erwachsenen zu unterscheiden.

\section{Gesunde Erwachsene}

Die 11. Variation der 6. Etüde von Paganini in der Klavier-Bearbeitung von Franz Liszt enthält zwei Abschnitte, die das Spielen von Tönen mit einer Geschwindigkeit von 1800 Tönen pro Minute bedingen. Eine solche Leistung benötigt sehr viel Übung. Ein Musiker hat bis zu seinem 18. Geburtstag sein Instrument rund $7500 \mathrm{~h}$ gespielt, was eine ungefähre Vorstellung von der benötigten Übung gibt. Ein Musikstück zu spielen gilt als eine der komplexesten menschlichen Leistungen. Für die Untersuchung der Neuroplastizität am hirngesunden Erwachsenen sind daher Musiker besonders geeignet: Das die Motorik der Hand betreffende Gebiet des Gyrus praecentralis war allgemein bei Berufsmusikern länger (d.h., die mit der Motorik der Hände befasste Gehirnmasse war grösser) als bei Nichtmusikern. Dieser Befund betraf bei Pianisten das entsprechende Areal beider Grosshirnhemisphären - im Unterschied $\mathrm{zu}$ Violinisten, bei denen lediglich das Areal der rechten Grosshirnhemisphäre eine Vergrösserung der Masse aufwies. Ein Pianist beansprucht die Motorik beider Hände sehr intensiv - im Unterschied zu einem Violinisten, der die Motorik hauptsächlich der linken Hand stark beansprucht (die linke Grosshirnhemisphäre steuert die Motorik der rechten Körperhälfte, die rechte die der linken) [4].

\section{KARGER}


Aus Tierversuchen ist bekannt, dass die graue Hirnmasse des Hippocampus in Abhängigkeit der Beanspruchung des Raumgedächtnisses variiert. In einer Untersuchung haben Maguire et al. [5] die Verhältnisse bei Londoner Taxi- und Busfahrern untersucht. Die betroffenen Gehirnareale wurden mittels Magnetresonanztomographie-basierter Morphometrie gemessen. Als Kontrollgruppe dienten Busfahrer, die eine gleich grosse Lenk-Erfahrung und eine gleich grosse Belastung mit Stress wie die Taxifahrer aufwiesen. Da sie einer vorgeschriebenen Route entlang fahren, ist ihr Raumgedächtnis nicht überdurchschnittlich beansprucht, im Unterschied zu den Taxifahrern, die in London unter anderem den Grundriss von über 25000 Strassen memorieren müssen. Die Taxifahrer wiesen eine grössere Masse in der hinteren Region des Hippocampus und eine kleinere in der vorderen Region auf. Die Autoren vermuten, dass die Zunahme der hinteren Masse zu Lasten der vorderen Masse geht, wo neue räumliche Gedächtnisinhalte verarbeitet werden.

Mittels funktioneller Magnetresonanztomographie (fMRI) untersuchten Maguire et al. [6], welche Gehirnareale beim Abrufen von Ereignissen aus der Lebensgeschichte (episodisches Gedächtnis) und welche beim Abrufen von Inhalten des Wissensgedächtnisses (semantisches Gedächtnis) aktiviert werden. (Die verschiedenen Gedächtnisformen sind an unterschiedlichen anatomischen Arealen des Grosshirns angeordnet [7].) Die Studienpopulation bestand aus 12 jungen Personen (mittleres Alter 32 Jahre) und 12 älteren Personen (mittleres Alter 75 Jahre). Die Prüfung des semantischen Gedächtnisses ergab keine Unterschiede, im Gegensatz zur Prüfung des episodischen Gedächtnisses: Bei den älteren Probanden waren der linke und der rechte Hippocampus aktiviert, bei den jungen Probanden lediglich der linke. Die Autoren vermuten, dass der linke Hippocampus im Verlauf des Lebens seine Kapazitätsgrenze erreicht, weil er nicht für die Länge des Lebens ausgelegt ist, die der heutige Mensch erreicht. Die Flexibilität des Gehirns bleibt aber erhalten, sodass einfach die Kapazität des brachliegenden rechten Hippocampus dazugeschaltet wird.

Leichtes aerobes Gehtraining führte bei älteren Erwachsenen zu einer Vergrösserung des Volumens sowohl des rechten auch des linken Hippocampus und Stretching zu einer Volumenabnahme beider Seiten. Die Volumina des Nucleus caudatus und des Thalamus blieben unverändert [8].

\section{Kinder, Jugendliche und ältere Erwachsene}

Aktivere Kinder mit einer besseren Kondition weisen unter anderem ein grösseres Volumen des Hippocampus und bessere kognitive Leistungen auf. Körperlich aktivere ältere Erwachsene mit einer guten Kondition weisen

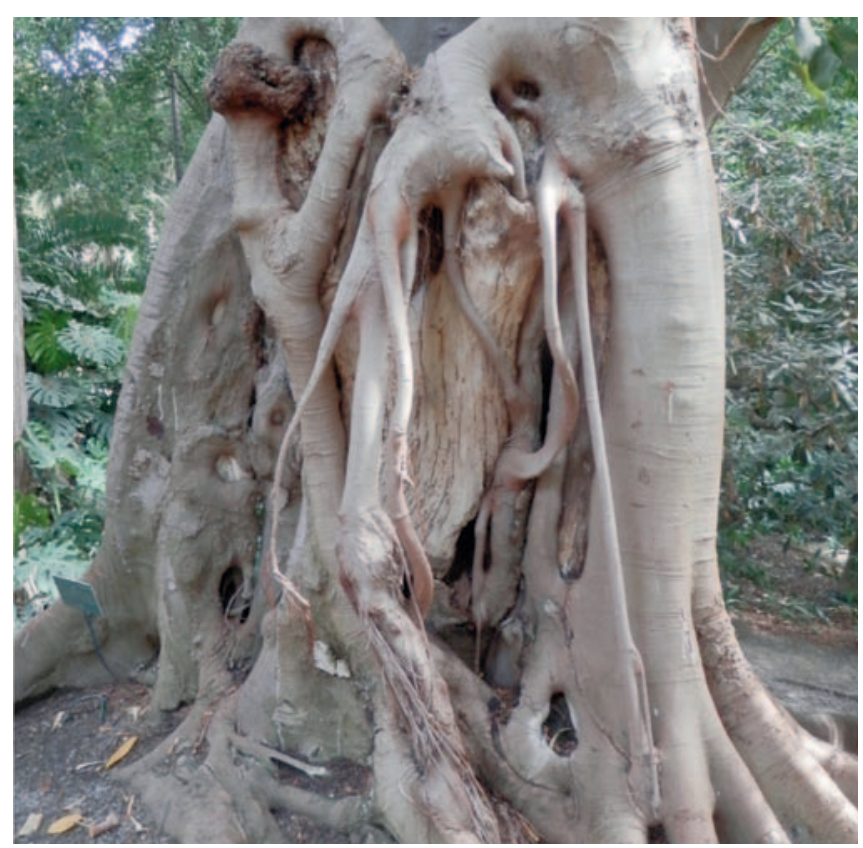

Abb. 2. Komplexes Wurzelwerk - eine Meisterleistung der Anpassung (Botanischer Garten, Malaga, 2017).

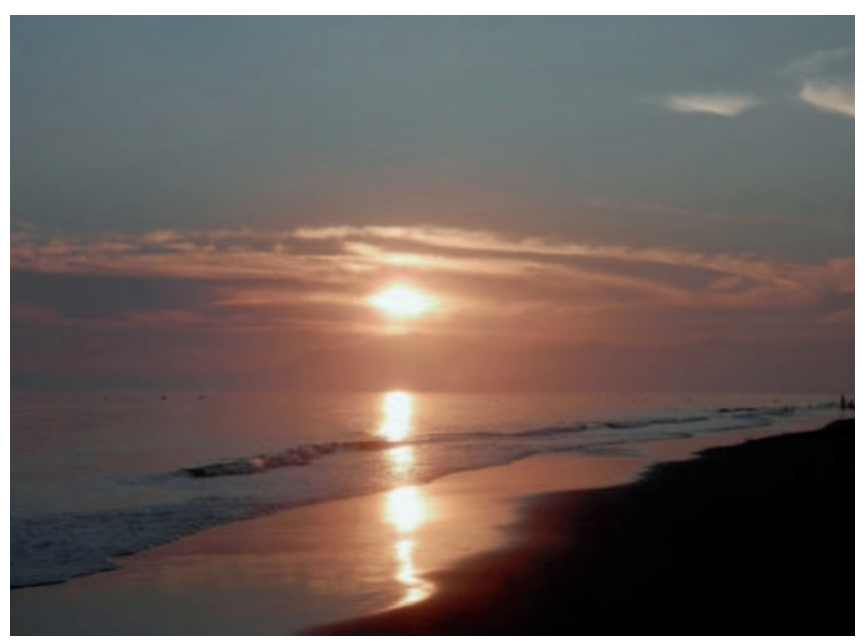

Abb. 3. Vier Mittel der physikalischen Therapie auf einem Bild: Licht, Luft, Wärme und Wasser (Strand in Marbella, 2017).

ebenfalls ein grösseres Volumen des Hippocampus, bessere Gedächtnisleistungen und eine bessere kognitive Kontrolle auf. Die Autoren halten es für erforderlich, den Beitrag von genetischen Faktoren und der Ernährung zu einer solchen Verbesserung zu untersuchen [9].

Im Tierversuch konnte gezeigt werden, dass aerobes Training die Blutversorgung der Grosshirnrinde intensiviert, die Anzahl der Synapsen vermehrt und die Entwicklung neuer Neurone fördert. Je besser trainiert, desto besser schnitten Versuchspersonen aus 2 Studien beim Testen der kognitiven Leistungen ab. Analoge Unterschiede konnten zwischen einer Interventionsgruppe, die ein ae- 
robes Training erhielt, und einer Kontrollgruppe, die kein solches Training erhielt, beobachtet werden. Mittels fMRI konnten die Effekte anatomisch dargestellt und quantifiziert werden. Eine gute Kondition und schon nur mässiges Training tragen durch ihre zentralnervöse Wirkung zu einer längeren Selbständigkeit im höheren Alter bei [2].

\section{Reflexionen}

Organe, die fleissig gebraucht werden, vergrössern ihre Masse und Leistung. Jedes Organ? Nein, das Nervengewebe und das Gehirn des Menschen führten einen erbitterten Kampf; sie wollten nicht dazugehören. Allein schon die Anatomie des Gehirns ist sehr komplex. Die Untersuchung von hirnverletzten Menschen und von ApoplexiePatienten ermöglichte ansatzweise eine Zuweisung ein- zelner Areale zu den entsprechenden Funktionen. Den Durchbruch brachten moderne bildgebende Verfahren wie die MRI. Das erlaubte, eine Struktur-Wirkungs-Beziehung herzustellen. Heutzutage ist es wissenschaftliches Allgemeinwissen, dass die Hirnstrukturen durch Bewegung, aber auch durch äussere Reize verändert werden können. Die Funktionen des Gehirns können systematisch trainiert werden; das Training äussert sich in Veränderungen der Struktur. Das ist, wie wenn ein Pneu dicker wird, je stärker er beansprucht wird. Das Gehirn ist auch sehr flexibel: Funktionen, die nicht gebraucht werden, werden abgeschaltet oder bei Bedarf auch zugeschaltet. Solches kann kein isoliert verabreichtes Medikament leisten, aber zum Beispiel die physikalische Therapie. Ganzheitliche Behandlungsansätze berücksichtigen die Einheit von Geist und Materie. Sie berücksichtigen beispielsweise auch soziale Aspekte.

\section{Literatur}

1 Colcombe SJ, et al: Cardiovascular fitness, cortical plasticity, and aging. PNAS 2004;101: 3316-3321.

2 Jeannin J-M: Physikalische Therapie: Neurotransmitter. Schweiz Z Ganzheitsmed 2017; 29:286-288.

3 Neurobiological effects of physical exercise. https://en.wikipedia.org/wiki/Neurobiological effects_of_physical_exercise (letzter Aufruf: 11.10.2017).
4 Jäncke L: The plastic human brain. Restor Neurol Neurosci 2009;27:521-538.

5 Maguire EA, et al: London taxi drivers and bus drivers: a structural MRI and neurospsychological analysis. Hippocampus 2006;16: 1091-1101.

6 Maguire EA, et al: Aging affects the engagement of the hippocampus during autobiographical memory retrieval. Brain 2003;126: 1511-1523.
7 Gedächtnis. https://de.wikipedia.org/wiki/ Ged\%C3\%A4chtnis (letzter Aufruf: 11.10.2017).

8 Erickson KI, et al: Exercise training increases size of hippocampus and improves memory. PNAS 2011;108:3017-3022.

9 Erickson KI, et al: Physical activity, brain, and cognition. Curr Opin Behav Sci 2015;4:27-32. 